\section{Remission nach AML stabilisieren}

Bei Patienten mit akuter myeloischer Leukämie (AML) in erster kompletter Remission, die nicht für eine allogene Stammzelltransplantation infrage kommen, reduziert die Kombination aus Histamindihydrochlorid (HDC, Ceple$n \mathrm{e}^{\circledast}$ ) und Interleukin 2 (IL-2) das Rezidivrisiko und hält die Remission stabil. Vermutlich wirken HDC und IL-2 synergistisch, so Gert Ossenkoppele, Amsterdam, Niederlande. Beide Substanzen schützen bzw. fördern die Funktion insbesondere von natürlichen Killerzellen und T-Zellen, welche für die immunvermittelte Zerstörung restlicher Leukämiezellen verantwortlich sind. Laut Fredrik Thorén, Göteborg, Schweden, ist diese Immuntherapie speziell bei den AML-Subtypen M4 und M5 sehr effektiv [Thorén FB et al. EHA 2011, Abstr. 056]. Die besonders hohe Exprimierung der NADPH-Oxidase durch Blasten mit Bildung radikaler Sauerstoffspezies wird durch die beiden Substanzen blockiert. Andreas Fischer

Nach Informationen von Meda Pharma

\section{Überlebensvorteil beim Prostatakarzinom}

Abirateronacetat (Zytiga ${ }^{\circledast}$ ) ist in Kombination mit Prednison/Prednisolon für die Therapie des metastasierten kastrationsresistenten Prostatakarzinom zugelassen, wenn die Erkrankung während oder nach einer Docetaxel-haltigen Chemotherapie progredient ist. Durch den sequenziellen Einsatz von Docetaxel (first-line) und Abirateronacetat mit Prednison/Prednisolon (second-line) verlängerte sich das mediane Überleben ab der ersten Docetaxel-Gabe auf 32,6 Monate [Goodman OB et al. J Clin Oncol. 2012;30(Suppl):Abstract 4558]. Mit Ausnahme der Patienten, die Docetaxel höchstens drei Monate erhielten, war der Überlebensvorteil durch die Addition von Abirateronacetat unabhängig vom Grund des Abbruchs der Docetaxel-Therapie und unabhängig von der Zeitspanne zwischen letzter Docetaxelund erster Abirateronacetat-Dosis. red

Nach Informationen von Janssen-Cilag

Immuntherapie des malignen Melanoms

\title{
Erfolge in der klinischen Praxis bestätigt
}

In einer Phase-III-Studie konnte erstmals ein verlängertes Überleben bei fortgeschrittenem malignen Melanom durch eine Immuntherapie erreicht werden.

Die mit Ipilimumab (Yervoy ${ }^{\circledR}$ ) behandelten Patienten zeigten einen statistisch signifikanten Überlebensvorteil von 3,7 Monaten im Vergleich zur Kontrollgruppe, die nur den Glykoprotein 100 (gp100)Peptid-Impfstoff erhalten hatte. Axel Hauschild, Kiel, wies auf die klinische Relevanz dieses Therapievorteils hin. Die 1-Jahres-Gesamtüberlebensrate betrug bei der Behandlung mit Ipilimumab 46\% unter Gabe von gp100 nur 24\%. Entsprechende Raten zum 2-Jahres-Überleben lagen bei 24 vs. $14 \%$ und auch die 3-Jahresdaten lassen laut Hauschild noch einen Vorteil erwarten.

Im Rahmen des Compassionate-UseProgramms erhielten laut Alexander Enk, Heidelberg, 185 Patienten Ipilimumab, von 161 Teilnehmern konnten Daten ausgewertet werden. Das klinische Ansprechen betrug 22,3\%, die 1-Jahres-Überlebensrate lag bei $36,4 \%$. Wirksamkeit und Sicherheit entsprachen auch in anderen Parametern den Angaben der Zulassungsstudie. Laut Enk ist das Ansprechen der Therapie oft erst nach Monaten zu beurteilen. Nebenwirkungen zwingen zwar mitunter zum Aussetzen der Therapie, deren Erfolg tritt dann aber dennoch oft später und auch anhaltend ein. Für Enk gehört die Therapie in die Erstlinientherapie der fortgeschrittenen inoperablen Melanomerkrankung. Die Zulassung sieht allerdings den Einsatz erst als Second-LineBehandlung vor.

Friederike Klein

Pressekonferenz „Ein Jahr mit Yervoy: Die Immuntherapie für Patienten mit vorbehandeltem metastasiertem Melanom" im Rahmen der 23. Fortbildungswoche für praktische Dermatologie und Venerologie, München, 23.07.2012; Veranstalter: Bristol-Myers Squibb

\section{„Continuation"-Erhaltungstherapie bei fortgeschrittenem NSCLC}

\section{Gesamtüberleben wurde verbessert}

Patienten mit fortgeschrittenem nicht-kleinzelligem Bronchialkarzinom (NSCLC) können von einer Erhaltungstherapie profitieren. Das bestätigen aktuelle Daten vom ASCO 2012.

Oft erhalten Patienten mit fortgeschrittenem NSCLC keine Zweitlinientherapie, weil sich ihr Allgemeinzustand seit der Erstlinientherapie infolge Krankheitsprogression verschlechtert. Mit einer Continuation-Erhaltungstherapie konnte in der PARAMOUNT-Studie erstmals das Gesamtüberleben verbessert werden. Bei der "Continuation"- wird im Unterschied zur „Switch"-Erhaltungstherapie mit einer Substanz weiterbehandelt, die bereits Bestandteil der Induktionstherapie war.

In dieser Studie erhielten 939 chemotherapienaive Patienten mit Nicht-Plat tenepithel-NSCLC im Stadium IIIB/IV eine Induktionstherapie mit vier Zyklen Cisplatin/Pemetrexed. Die 539 Patienten, die ein objektives Ansprechen oder eine Stabilisierung erreicht hatten, erhielten randomisiert eine Erhaltungstherapie entweder mit Pemetrexed (Alimta ${ }^{\circ}$ ) oder Placebo (jeweils zur optimalen Supportivtherapie). Die Pemetrexed-Erhaltungstherapie verlängerte das progressionsfreie Überleben (primärer Endpunkt) signifikant auf 4,1 vs. 2,8 Monate ( $p=0,00006)$ [Paz-Ares LG et al. J Clin Oncol. 2011; 29(Suppl):abstr. CRA7510]. Aktuellen Daten zufolge lebten die Patienten im Pemetrexed-Erhaltungsarm auch signifikant länger (13,9 vs. 11,0 Monate, $\mathrm{p}=0,0196$ ) [Paz-Ares LG et al. J Clin Oncol. 2012; 30(Suppl):abstr. LBA7507]. Alle untersuchten Subgruppen profitierten von der Erhaltungstherapie.

Günther Springer

Presse-Roundtable „Lilly Post-ASCO Update: Neue Daten zur NSCLC-Erhaltungstherapie mit Pemetrexed", 04.07.2012, Frankfurt/Main; Veranstalter: Lilly 